\title{
Success Run Waiting Times and Fuss-Catalan Numbers
}

\author{
S. J. Dilworth ${ }^{1}$ and S. R. Mane ${ }^{2}$ \\ ${ }^{1}$ Department of Mathematics, University of South Carolina, Columbia, SC 29208, USA \\ ${ }^{2}$ Convergent Computing Inc., P.O. Box 561, Shoreham, NY 11786, USA
}

Correspondence should be addressed to S. R. Mane; srmane001@gmail.com

Received 10 June 2015; Accepted 3 September 2015

Academic Editor: Steve Su

Copyright (C) 2015 S. J. Dilworth and S. R. Mane. This is an open access article distributed under the Creative Commons Attribution License, which permits unrestricted use, distribution, and reproduction in any medium, provided the original work is properly cited.

\begin{abstract}
We present power series expressions for all the roots of the auxiliary equation of the recurrence relation for the distribution of the waiting time for the first run of $k$ consecutive successes in a sequence of independent Bernoulli trials, that is, the geometric distribution of order $k$. We show that the series coefficients are Fuss-Catalan numbers and write the roots in terms of the generating function of the Fuss-Catalan numbers. Our main result is a new exact expression for the distribution, which is more concise than previously published formulas. Our work extends the analysis by Feller, who gave asymptotic results. We obtain quantitative improvements of the error estimates obtained by Feller.
\end{abstract}

\section{Introduction}

For a sequence of independent Bernoulli trials with probability $p$ of success, let $N_{k}$ be the waiting time for the first run of $k$ consecutive successes. Then $N_{k}$ is said to have the geometric distribution of order $k$. See, for example, the texts by Balakrishnan and Koutras [1] and by Johnson et al. [2]. An expression for $f_{k}$ (the probability mass function of $N_{k}$ ) was derived by Philippou et al. [3] in terms of multinomial sums, following earlier work by Philippou and Muwafi [4]. By a clever counting argument, Burr and Cane [5] obtained an expression for $P\left(N_{k}>n\right)$ as a sum of $O\left(n^{2} / k\right)$ terms involving products of two binomial coefficients (rederived by Godbole [6] using a different method). A somewhat different expression for $P\left(N_{k}>n\right)$ involving multinomial coefficients was obtained by Philippou and Makri [7]. Perhaps the simplest expression to date is the one by Muselli [8, eq. 16], which requires a sum over $1+\lfloor(n+1) /(k+1)\rfloor$ binomial coefficients.

In his classic text [9, pp. 322-326], Feller took a different approach to the distribution of $N_{k}$ by setting up a suitable recurrence relation. The auxiliary equation has degree $k$. Feller showed that the equation has a unique largest (in absolute value) "principal root," which is real and positive, and hence obtained an asymptotic formula for $f_{k}$ in terms of the principal root (more precisely, we work with $z \in \mathbb{C}$ (see Section 2) but Feller employed $s=1 / z$; hence in Feller's formalism the principal root has the smallest absolute value). Feller also bounded the error from neglecting the contribution from the other roots and formulated an iteration scheme for approximating the principal root numerically. Concerning the formula for the exact solution given by the recurrence relation method, Feller commented that it was "primarily of theoretical interest" because "the labor involved in computing all the roots is usually prohibitive" [9, p. 276]. Feller's work dates from 1968 and computing power for numerical analysis has increased greatly since then. Note, however, that our derivation below is purely analytical.

We extend Feller's analysis [9] of the recurrence relation for $f_{k}$ by finding power series expressions for all the roots of the auxiliary equation. We show that the series coefficients are Fuss-Catalan numbers (see the text by Graham et al. [10]) and the roots are given by suitable values of the generating function of the Fuss-Catalan numbers. This permits the roots to be written in terms of known "elementary functions." This leads to our main result (21) which is a new exact expression for $P\left(N_{k}>n\right)$. This formula differs from the other results mentioned above in the important respect that there are only $k$ summands (independently of $n$ ). We also obtain quantitative improvements of the error estimates obtained by Feller. We also derive numerous properties of the roots of the auxiliary equation; for example, we draw attention to the fact 
that, in many results below, the value $p=k /(k+1)$ is a special case, and the ranges $0<p<k /(k+1)$ and $k /(k+1)<p<1$ require separate treatments.

Our analysis below focuses only on the original problem treated by Feller. More recent papers study variants of the problem; for example, Eryilmaz [11] treats the geometric distribution of order $k$ with a reward, where each time a success occurs a random reward is received, while Shmerling [12] studies a generalization of the geometric distribution of order $k$ for Markov processes.

\section{Notation and Definitions}

2.1. Auxiliary Equation. We present our basic notation and definitions below. The probability mass function $f_{k}$ of $X$ satisfies the recurrence relation, for $n>k$,

$$
\begin{aligned}
f_{k}(n)= & q f_{k}(n-1)+p q f_{k}(n-2)+p^{2} q f_{k}(n-3) \\
& +\cdots+p^{k-1} q f_{k}(n-k) .
\end{aligned}
$$

Here and below we define $q=1-p$. A derivation of (1) was given by Barry and Lo Bello [13]. We employ the initial conditions $f_{k}(n)=0$ for $n=1, \ldots, k-1$ and $f_{k}(k)=p^{k}$. Next we define the auxiliary polynomial

$$
\begin{aligned}
\mathscr{A}_{p}(z)= & z^{k}-q z^{k-1}-q p z^{k-2}-q p^{2} z^{k-3}-\cdots \\
& -q p^{k-1} .
\end{aligned}
$$

The auxiliary equation is $\mathscr{A}_{p}(z)=0$. We shall drop the subscript " $p$ " unless necessary.

To establish contact with Feller's formalism [9], note that he derived the following expression for the probability generating function $[9$, eq. (7.6)]:

$$
\begin{aligned}
\mathscr{P}(s) & =\frac{p^{k} s^{k}(1-p s)}{1-s+q p^{k} s^{k+1}} \\
& =\frac{p^{k} s^{k}}{1-q s\left(1+p s+\cdots+p^{k-1} s^{k-1}\right)} .
\end{aligned}
$$

Feller then considered the roots of the polynomial in the denominator of (3). Setting $z=1 / s$ in (2), this is equivalent to our auxiliary polynomial

$$
s^{k} \mathscr{A}\left(\frac{1}{s}\right)=1-q s\left(1+p s+\cdots+p^{k-1} s^{k-1}\right) .
$$

We shall employ $z$ and (2) below, bearing in mind throughout that Feller worked with $s=1 / z$.

Feller [9] proved that the roots of the auxiliary equation are distinct. We denote the roots by $\lambda_{j}(p, k), j=0,1, \ldots, k-1$. Unless required, we shall omit the arguments $p$ and $k$. It is useful to multiply $\mathscr{A}(z)$ by $(z-p)$ to obtain the polynomial

$$
\mathscr{B}(z)=(z-p) \mathscr{A}(z)=z^{k}(z-1)+p^{k}(1-p) .
$$

It was shown by Feller [9] that there is exactly one real root for $z \in(0,1)$. This real positive root will feature sufficiently prominently in our analysis below that we designate it by the symbol $\alpha$ and call it the "principal root." The other roots will be termed "secondary roots." We define $\lambda_{0}=\alpha$. For brevity in various calculations below, we also define $p_{*}=k /(k+1)$ and $q_{*}=1 /(k+1)$.

2.2. Special Cases $p=0$ and $p=1$. The special cases $p=0$ and $p=1$ are usually ignored in the literature, but it is useful to record the solutions, to help with limits for other calculations for $p \in(0,1)$. Clearly if $p=0$ then $f_{k}(n)=0$ for all $n$. Also if $p=1$ then $f_{k}(k)=1$ and $f_{k}(n)=0$ for all other values of $n$. However, we are more interested in the roots of the auxiliary equation. If $p=1$ the auxiliary equation is $z^{k}=0$ and all the roots vanish. If $p=0$ the auxiliary equation is $z^{k-1}(z-1)=0$. One root is $z=1$ and there are $k-1$ repeated roots $z=0$. Hence $\alpha=1$ if $p=0$ and $\alpha=0$ if $p=1$ while in both cases $\lambda_{j}=0$ for $j=1, \ldots, k-1$. These facts will be helpful below.

\section{Main Results}

Theorem 1 (roots of auxiliary equation). For $n \geq 1$, let

$$
b_{n}=\frac{\Gamma(n-1+n / k)}{n ! \Gamma(n / k)} \text {. }
$$

For $p \in(0,1)$, the secondary roots are given by

$$
\lambda_{j}=\sum_{n=1}^{\infty} b_{n}\left(e^{2 \pi i j / k} p q^{1 / k}\right)^{n}, \quad(1 \leq j<k) .
$$

The principal root $\alpha$ is given by

$$
\alpha= \begin{cases}1-k \sum_{m=1}^{\infty} b_{m k}\left(p^{k} q\right)^{m} & p<\frac{k}{(k+1)}, \\ \frac{k}{(k+1)} & p=\frac{k}{(k+1)}, \\ \sum_{n=1}^{\infty} b_{n}\left(p q^{1 / k}\right)^{n} & p>\frac{k}{(k+1)} .\end{cases}
$$

The derivation of the above expressions will be given in Section 5. Feller [9] proved that there is a unique real positive root and that its magnitude exceeds that of all the other roots.

Remark 2 (Fuss-Catalan numbers). Relevant definitions, formulas, and identities for the Fuss-Catalan numbers can be found in the text by Graham et al. [10]. The Fuss-Catalan numbers are

$$
A_{m}(\nu, r)=\frac{r}{\Gamma(m+1)} \frac{\Gamma(m \nu+r)}{\Gamma(m(\nu-1)+r+1)} .
$$

The numbers are well defined provided $m v+r \neq 0$. Hence the coefficients $b_{n}$ defined in (6) are

$$
b_{n}=-A_{n}\left(1+\frac{1}{k},-1\right) .
$$


The generating function of the Fuss-Catalan numbers is $B_{v}(z)$ and $[10$, p. 363$]$

$$
\begin{gathered}
B_{\nu}(z)=\sum_{m=0}^{\infty} A_{m}(\nu, 1) z^{m}, \\
B_{\nu}(z)^{r}=\sum_{m=0}^{\infty} A_{m}(\nu, r) z^{m} .
\end{gathered}
$$

Hence for all $0<p<1$, the secondary roots are given by

$$
\begin{aligned}
\lambda_{j} & =1-\sum_{n=0}^{\infty} A_{n}\left(1+\frac{1}{k},-1\right)\left(e^{2 \pi i j / k} p q^{1 / k}\right)^{n} \\
& =1-\frac{1}{B_{1+1 / k}\left(e^{2 \pi i j / k} p q^{1 / k}\right)}, \quad(1 \leq j<k) .
\end{aligned}
$$

For $k /(k+1)<p<1$, the above expression also applies to $\alpha=\lambda_{0}$. For $0<p<k /(k+1)$, note that

$$
\begin{aligned}
k b_{m k} & =k \frac{\Gamma(m(k+1)-1)}{(m k) ! \Gamma(m)}=\frac{(m(k+1)-2) !}{(m k-1) ! m !} \\
& =-A_{m}(k+1,-1) .
\end{aligned}
$$

Hence for $0<p<k /(k+1)$,

$$
\alpha=\sum_{m=0}^{\infty} A_{m}(k+1,-1)\left(p^{k} q\right)^{m}=\frac{1}{B_{k+1}\left(p^{k} q\right)} .
$$

This establishes the connection of the roots of the auxiliary equation to the generating functions of the Fuss-Catalan numbers.

Theorem 3 (probability mass function). For $n>k$, the probability mass function is given by

$$
\begin{aligned}
& f_{k}(n) \\
& \quad= \begin{cases}\frac{p^{k}}{k+1} \sum_{j=0}^{k-1} \lambda_{j}^{n-k} \frac{\lambda_{j}-p}{\lambda_{j}-k /(k+1)}, & p \neq \frac{k}{k+1}, \\
\frac{p^{k}}{k+1}\left(2 \alpha^{n-k}+\lambda_{1}^{n-k}+\cdots+\lambda_{k-1}^{n-k}\right) & p=\frac{k}{k+1} .\end{cases}
\end{aligned}
$$

The proof will be given in Section 6 .

Corollary 4 (asymptotic solution). For fixed $k$ and $n \gg k$, the contribution to $f_{k}(n)$ is dominated by the principal root, and so asymptotically

$$
f_{k}(n) \asymp \begin{cases}\alpha^{n} \frac{(1-\alpha)(\alpha-p)}{((k+1) \alpha-k) q}, & p \neq \frac{k}{(k+1)}, \\ \frac{2 p^{n}}{k+1} & p=\frac{k}{(k+1)} .\end{cases}
$$

The expression for $p \neq k /(k+1)$ was derived by Feller [9]. We formulate the notion of "asymptotic" more precisely as follows. For fixed $\epsilon>0$, we demand that the magnitude of the contribution to $f_{k}(n)$ in (15) from all the secondary roots is less than $\epsilon$ times the contribution from the principal root. This is achieved if $n \geq N(p, k)$, where

$$
N=k+\frac{\ln (\epsilon /(k-1))}{\ln \kappa} .
$$

Here

$$
\kappa= \begin{cases}\frac{p}{(1-p)} & p \in\left(0, \frac{1}{(k+1)}\right], \\ \min \left\{\frac{p(k+1)}{k}, 1-\delta\right\} & p \in\left(\frac{1}{(k+1)}, \frac{k}{(k+1)}\right), \\ 1-\delta & p \in\left[\frac{k}{(k+1)}, 1\right) .\end{cases}
$$

Here

$$
\begin{aligned}
\delta & =\frac{1}{(k+1)^{1 / k}}\left[1+\frac{p q^{1 / k}}{k}\right. \\
& \left.-\sqrt{1+\frac{\left(p q^{1 / k}\right)^{2}}{k^{2}}+\frac{2 p q^{1 / k}}{k} \cos \left(\frac{2 \pi}{k}\right)}\right] .
\end{aligned}
$$

The derivations of the expressions for $N(p, k), \kappa$, and $\delta$ will be given in Section 7 .

Theorem 5 (probability of longest run). Let $P\left(L_{n}<k\right)$ denote the probability that, in a sequence of $n$ Bernoulli trials, the longest run of successes has length less than $k$. Note that $P\left(N_{k}>\right.$ $n)=P\left(L_{n}<k\right)$. Then

$$
f_{k}(n+k+1)=p^{k} q P\left(L_{n}<k\right) .
$$

Hence

$$
\begin{aligned}
& P\left(L_{n}<k\right) \\
& \quad= \begin{cases}\frac{1}{(k+1) q} \sum_{j=0}^{k-1} \lambda_{j}^{n+1} \frac{\lambda_{j}-p}{\lambda_{j}-k /(k+1)} & p \neq \frac{k}{(k+1)}, \\
2 \alpha^{n+1}+\lambda_{1}^{n+1}+\cdots+\lambda_{k-1}^{n+1} & p=\frac{k}{(k+1)} .\end{cases}
\end{aligned}
$$

An expression for $P\left(L_{n}<k\right)$ was derived by Muselli [8, eq. 16] and requires a sum of $1+\lfloor(n+1) /(k+1)\rfloor$ terms; namely,

$$
\begin{aligned}
& P\left(L_{n}<k\right)=\sum_{m=0}^{\lfloor(n+1) /(k+1)\rfloor}(-1)^{m} \\
& \cdot p^{m k} q^{m-1}\left(\left(\begin{array}{c}
n-m k \\
m-1
\end{array}\right)+q\left(\begin{array}{c}
n-m k \\
m
\end{array}\right)\right) .
\end{aligned}
$$

Expressions involving (possibly nested) sums of binomial or multinomial coefficients were derived by Burr and Cane [5] 
and Philippou and Makri [7]. For fixed $k$, our formula (21) requires a sum of exactly $k$ terms, independently of $n$ (this can be reduced to $\lfloor(k+1) / 2\rfloor$ terms by noting that the complex roots occur in conjugate pairs: there are $k-1$ (resp., $k-2$ ) complex roots for odd (resp., even) $k$ ).

Corollary 6 (asymptotic solution). For fixed $k$ and $n \gg k$, the contribution to $P\left(L_{n}<k\right)$ is dominated by the principal root, and so asymptotically

$$
P\left(L_{n}<k\right) \asymp \begin{cases}\alpha^{n+1} \frac{\alpha-p}{((k+1) \alpha-k) q} & p \neq \frac{k}{(k+1)}, \\ 2 p^{n+1} & p=\frac{k}{(k+1)} .\end{cases}
$$

The asymptotic solution for $p \neq k /(k+1)$ was derived by Feller [9]. The identity in (20) is obvious, which yields (21) immediately from (15). Similarly, the asymptotic expressions in (23) follow from (16).

\section{Properties of Roots}

Section 4.1 presents results which are essential to prove the main results of our paper in Sections 5, 6, and 7. Section 4.2 contains additional results, which can be omitted by the reader who wishes to proceed directly to Section 5 . We rewrite the equation $\mathscr{B}(z)=0$ in the form

$$
z^{k}(1-z)=p^{k}(1-p) .
$$

We assume $p \in(0,1)$ below; expressions where $p=0$ or 1 will be indicated as appropriate.

\subsection{Properties of Roots I}

Remark 7. Feller [9] proved that all the roots of the auxiliary equation are distinct. We include a summarized proof for completeness (see also Barry and Lo Bello [13]). Recall that $\mathscr{B}(z)$ has all the roots of $\mathscr{A}(z)$ and an extra root $z=p$. Now

$$
\begin{aligned}
\mathscr{B}^{\prime}(z) & =(k+1) z^{k}-k z^{k-1} \\
& =(k+1) z^{k-1}\left[z-\frac{k}{k+1}\right] .
\end{aligned}
$$

Then $\mathscr{B}^{\prime}\left(z_{*}\right)=0$ when $z_{*}=0$, which is not a root of $\mathscr{B}(z)$, or else $z_{*}=k /(k+1)$. But $z_{*}=k /(k+1)$ is a root of $\mathscr{B}(z)$ if and only if $p=k /(k+1)$; that is, $z_{*}=p=k /(k+1)$. So $\mathscr{B}(z)$ can have a repeated root (of order 2 ) only when $p=k /(k+1)$. The roots are at $z_{*}=p$ and we know that one of those two roots is not a root of $\mathscr{A}(z)$. Hence $\mathscr{A}(z)$ has no repeated roots.

Proposition 8. For $p \in(0,1)$, the auxiliary equation has a unique positive real root, which lies in $(0,1)$. One denotes the positive real root by $\alpha$. For any $p \in(0,1)$, exactly one of the three following statements is true:
(i) $0<p<k /(k+1)<\alpha<1$.
(ii) $0<\alpha<k /(k+1)<p<1$.
(iii) $\alpha=p=k /(k+1)$.

Proof. It was proved by Feller [9], who employed $s=1 / z$, that the auxiliary equation has a unique positive real root. In Feller's analysis, the root had a magnitude larger than unity, so in our case the root lies in $(0,1)$. The mutually exclusive statements (i), (ii), and (iii) follow immediately from an examination of the level sets of $p^{k}(1-p)$ for $p \in(0,1)$, bearing in mind that $(24)$ has a repeated root of order 2 when $p=k /(k+1)$.

Proposition 9 (Feller [9]). For $p \in(0,1)$, the principal root $\alpha$ has a strictly greater magnitude than all the other roots of the auxiliary equation; that is, $0<\left|z_{r}\right|<\alpha<1$, where $z_{r} \in \mathbb{C} \backslash \alpha$ is a root of $\mathscr{A}(z)$. One employs the term "secondary roots" for the set $\left\{\lambda_{j}, j=1, \ldots, k-1\right\}$.

Proof. This was proved by Feller [9], who employed $s=$ $1 / z$ and showed that the positive real root has a smaller magnitude (in his case) than all the other roots.

Proposition 10. Suppose $p_{1}, p_{2} \in(0,1)$ and $p_{1} \neq p_{2}$ and $p_{1}^{k}\left(1-p_{1}\right)=p_{2}^{k}\left(1-p_{2}\right)$. Writing $\alpha(p)$ to denote the dependence on $p$, then $p_{2}=\alpha\left(p_{1}\right)$ and $p_{1}=\alpha\left(p_{2}\right)$. Also the secondary roots of the auxiliary polynomials $\mathscr{A}_{p_{1}}(z)$ and $\mathscr{A}_{p_{2}}(z)$ are identical; that is, $\lambda_{j}\left(p_{1}\right)=\lambda_{j}\left(p_{2}\right)$ for $j=1, \ldots, k-1$.

Proof. Clearly the equations $z^{k}(1-z)=p_{1}^{k}\left(1-p_{1}\right)$ and $z^{k}(1-$ $z)=p_{2}^{k}\left(1-p_{2}\right)$ are identical. Hence the equations have the same roots. Since both equations have exactly two positive roots, which are $\left\{p_{1}, \alpha\left(p_{1}\right)\right\}$ and $\left\{p_{2}, \alpha\left(p_{2}\right)\right\}$, respectively, and $p_{1} \neq p_{2}$, it follows that $p_{2}=\alpha\left(p_{1}\right)$ and $p_{1}=\alpha\left(p_{2}\right)$. The secondary roots of the two equations are obviously identical.

It follows that $\alpha(\alpha(p))=p$ for any $p \in(0,1)$. From above, if $p \neq k /(k+1)$ then $\alpha(\alpha(p))=p$ and if $p=k /(k+1)$, then $p=\alpha(p)=k /(k+1)$, which proves the result.

Proposition 11. Let $p \in(0,1)$. The principal root $\alpha$ satisfies the bounds $\alpha>q$ and $\alpha<1-p^{k} q$.

Proof. The bound $\alpha>q$ follows because

$$
\begin{aligned}
\mathscr{A}(q) & =q^{k}-q^{k}-p q^{k-1}-\cdots-p^{k-1} q=-\sum_{j=1}^{k-1} p^{j} q^{k-j} \\
& <0 .
\end{aligned}
$$

Then because $\mathscr{A}(0)<0, \mathscr{A}(1)>0$ and because $\alpha$ is the unique positive root, it follows that $0<q<\alpha$. The upper bound $\alpha=1-p^{k} q$ is derived as follows:

$$
\alpha=1-\frac{p^{k}(1-p)}{\alpha^{k}}<1-q p^{k} .
$$

These bounds are not tight; for example, if $p=k /(k+1)$ then $\alpha=k /(k+1)$ but $q=1 /(k+1)$ so if $k \gg 1$ then $\alpha \gg q$. 


\subsection{Properties of Roots II}

Proposition 12. For $0<p<1$, the roots are continuously differentiable functions of $p$.

Proof. We have seen that the roots are distinct. In this section we write $\mathscr{A}(z, p)$ and $\mathscr{B}(z, p)$ and employ subscripts " $z$ " and " $p$ " below to denote partial derivatives. We show that if $z_{r}$ is a root, $\left(\mathscr{A}_{z}, \mathscr{A}_{p}\right)_{z=z_{r}} \neq(0,0)$. Note that

$$
\begin{aligned}
& \mathscr{B}_{z}(z, p)=z^{k-1}((k+1) z-k), \\
& \mathscr{B}_{p}(z, p)=p^{k-1}(k-(k+1) p) .
\end{aligned}
$$

The former vanishes only at $z=0$ (not a root) or $z=k /(k+1)$, which is a root only if $p=k /(k+1)$. For $0<p<1$, the latter vanishes only at $p=k /(k+1)$. Next

$$
\begin{aligned}
& \mathscr{B}_{z}(z, p)=(z-p) \mathscr{A}_{z}(z, p)+\mathscr{A}(z, p), \\
& \mathscr{B}_{p}(z, p)=(z-p) \mathscr{A}_{p}(z, p)-\mathscr{A}(z, p) .
\end{aligned}
$$

Hence at a root $z_{r}, \mathscr{B}_{z}\left(z_{r}, p\right)=\left(z_{r}-p\right) \mathscr{A}_{z}\left(z_{r}, p\right)$ and $\mathscr{B}_{p}\left(z_{r}, p\right)=\left(z_{r}-p\right) \mathscr{A}_{p}\left(z_{r}, p\right)$. Hence $\left(\mathscr{A}_{z}, \mathscr{A}_{p}\right) \neq(0,0)$ for all the secondary roots. For the primary root $\alpha$, if $p \neq k /(k+1)$ then $z_{r}=\alpha \neq p$ and so $\left(\mathscr{A}_{z}, \mathscr{A}_{p}\right) \neq(0,0)$. If $p=k /(k+1)$ then $\alpha=p$. We treat this case as follows. First, the partial derivatives are

$$
\begin{aligned}
& \mathscr{A}_{z}(z, p) \\
& =k z^{k-1} \\
& \quad-q\left[(k-1) z^{k-2}+(k-2) p z^{k-3}+\cdots+p^{k-2}\right], \\
& \mathscr{A}_{p}(z, p) \\
& =z^{k-1}+p z^{k-2}+\cdots+p^{k-1} \\
& \quad-q\left[z^{k-2}+2 p z^{k-3}+\cdots+(k-1) p^{k-2}\right] .
\end{aligned}
$$

Now put $z=p=k /(k+1)$; then

$$
\begin{aligned}
\mathscr{A}_{z}(p, p)= & k p^{k-1} \\
& -q p^{k-2}[(k-1)+(k-2)+\cdots+1] \\
= & \frac{1}{2} k p^{k-2}, \\
\mathscr{A}_{p}(p, p)= & k p^{k-1}-q p^{k-2}[1+2+\cdots+(k-1)] \\
= & \frac{1}{2} k p^{k-2} .
\end{aligned}
$$

Hence $\left(\mathscr{A}_{z}, \mathscr{A}_{p}\right) \neq(0,0)$ for $\alpha=p=k /(k+1)$. The implicit function theorem then yields that every root $z_{r}$ is a continuously differentiable function of $p$.
Proposition 13. (a) The principal root $\alpha$ decreases monotonically and continuously from 1 to 0 as p increases from 0 to 1 .

(b) If $k$ is odd, there are no other real roots. If $k$ is even, there is exactly one real negative root.

(c) All the other roots are complex and form conjugate pairs. There are no pure imaginary roots.

Proof. (a) This is obvious by examining the level sets of $p^{k}(1-$ $p)$. For any $p \in(0,1)$, the level set is $\{p, \alpha\}$. As $p$ increases then $\alpha$ decreases monotonically and continuously, and we have seen $\alpha=1$ for $p=0$ and $\alpha=0$ for $p=1$. (b) If $k$ is odd, then $z^{k}(1-z)<0$ for $z<0$ and there are no other real roots. If $k$ is even, then $z^{k}(1-z)>0$ for $z<0$ and increases continuously without bound as $z \rightarrow-\infty$. Hence there is exactly one real negative root. (c) Obviously all the other roots are complex, and they form complex conjugate pairs because the coefficients of the auxiliary equation are real. To show there are no pure imaginary roots, let $z=i y$, where $y$ is real. Then for any integer $k, z^{k}(1-z)=i^{k} y^{k}(1-i y)$ is never real; hence it cannot equal $p^{k}(1-p)$; hence $z$ is not a root.

Proposition 14. For any $p \in(0,1)$, the secondary roots $\lambda_{j}(p)$, $j=1, \ldots, k-1$, satisfy the inequality

$$
\begin{aligned}
0 & <\left|\lambda_{j}(p)\right|<\min \{p, \alpha(p)\} \leq \frac{k}{k+1} \\
& \leq \max \{p, \alpha(p)\}<1 .
\end{aligned}
$$

The inequalities involving $k /(k+1)$ are strict if $p \neq k /(k+1)$. This is a stronger bound than Proposition 9.

Proof. We already know that $p=\alpha(p)$ if and only if $p=$ $k /(k+1)$. For the secondary roots, we proved $\left|\lambda_{j}(p)\right|<\alpha(p)$. Hence $\left|\lambda_{j}(p)\right|=\left|\lambda_{j}(\alpha(p))\right|<\alpha(\alpha(p))=p$ which yields the claimed inequality.

Proposition 15. For $p \in(0,1)$, let $R(p)$ denote the set of $k+1$ roots of the equation $z^{k}(1-z)=p^{k}(1-p)$. Let $p_{1}, p_{2} \in(0,1)$. Then $R\left(p_{1}\right)=R\left(p_{2}\right)$ if $p_{2}=p_{1}$ or $p_{2}=\alpha\left(p_{1}\right)$; otherwise $R\left(p_{1}\right) \cap R\left(p_{2}\right)=\varnothing$.

Proof. Suppose $R\left(p_{1}\right) \cap R\left(p_{2}\right) \neq \varnothing$. Let $z \in R\left(p_{1}\right) \cap R\left(p_{2}\right)$. Then $z^{k}(1-z)=p_{1}^{k}\left(1-p_{1}\right)=p_{2}^{k}\left(1-p_{2}\right)$. Hence either $p_{2}=p_{1}$ or $p_{2}=\alpha\left(p_{1}\right)$, in which case $R\left(p_{1}\right)$ and $R\left(p_{2}\right)$ coincide; else $z$ does not exist and $R\left(p_{1}\right) \cap R\left(p_{2}\right)=\varnothing$.

Proposition 16. Let $p \in(0,1)$ and let $z_{1}$ and $z_{2}$ be two distinct roots. Then

(a) $\left|z_{1}\right|=\left|z_{2}\right|$ if and only if $z_{1}=\bar{z}_{2}$.

(b) $\mathfrak{R}\left(z_{1}\right)<\mathfrak{R}\left(z_{2}\right)$ if and only if $\left|z_{1}\right|<\left|z_{2}\right|$.

(c) For even $k$, the negative real root has the most negative real part, hence the smallest amplitude of all the roots.

Proof. (a) Suppose $\left|z_{1}\right|=\left|z_{2}\right|$. Then $\left|z_{1}\right|^{k}\left|1-z_{1}\right|=\left|z_{2}\right|^{k} \mid 1-$ $z_{2} \mid=p^{k}(1-p)$. Cancelling the factors of $\left|z_{1}\right|^{k}$ and $\left|z_{2}\right|^{k}$ and squaring yields $\left|1-z_{1}\right|^{2}=\left|1-z_{2}\right|^{2}$, so

$$
1-2 \Re\left(z_{1}\right)+\left|z_{1}\right|^{2}=1-2 \Re\left(z_{2}\right)+\left|z_{2}\right|^{2} .
$$


Cancel terms to deduce $\mathfrak{R}\left(z_{1}\right)=\mathfrak{R}\left(z_{2}\right)$. But since $\left|z_{1}\right|=\left|z_{2}\right|$ this implies $\mathfrak{\Im}\left(z_{1}\right)= \pm \mathfrak{I}\left(z_{2}\right)$. Hence either $z_{1}=z_{2}$ or $z_{1}=$ $\bar{z}_{2}$. Geometrically, the condition $\left|z_{1}\right|=\left|z_{2}\right|$ implies equal distance from origin (circle around $z=0$ ), and $\left|1-z_{1}\right|=$ $\left|1-z_{2}\right|$ implies equal distance from $z=1$ (circle around $z=1$ ). The solutions are the points of intersection of the two circles that is symmetric around the real axis, hence complex conjugates. (b) Note that $\bar{z}^{k}(1-\bar{z})=z^{k}(1-z)=q p^{k}$. Multiply to obtain $|z \bar{z}|^{k}\left(1-z-\bar{z}+|z|^{2}\right)=q^{2} p^{2 k}$. Then $z \neq 0$ so rearrange terms to obtain

$$
2 \Re(z)=1+|z|^{2}-\frac{q^{2} p^{2 k}}{|z|^{2 k}} .
$$

All the roots satisfy this equation. The right-hand side of (34) is a continuous monotonically increasing function of $|z|$. Hence if $z_{1}$ and $z_{2}$ are two roots with unequal amplitudes then $\left|z_{1}\right|<\left|z_{2}\right|$ implies $\mathfrak{R}\left(z_{1}\right)<\mathfrak{R}\left(z_{2}\right)$ and vice versa. (c) For even $k$, let $z_{2}$ in (b) be real and negative. Suppose a root $z_{1}$ exists such that $\mathfrak{R}\left(z_{1}\right)<\mathfrak{R}\left(z_{2}\right)$; then necessarily $\left|z_{1}\right|>\left|z_{2}\right|$, which contradicts the result in (b). Hence the negative real root has the most negative real part and the smallest amplitude of all the roots.

We proved in (32) that $\left|\lambda_{j}\right|<\min \{p, \alpha\}$ for all the secondary roots. We also know that all the secondary roots vanish, that is, attaining their minimum amplitudes, at $p=0$ or 1 .

Proposition 17. All the secondary roots simultaneously attain their maximum amplitudes at $p=k /(k+1)$.

Proof. Let $z_{j}$ be a secondary root. For all $p \in(0,1)$, we know from (7) that $z_{j}$ is a series in powers of $e^{2 \pi i j / k} p q^{1 / k}$; hence $z_{j}$ is differentiable with respect to $p$ within the radius of convergence of that series. We drop the subscript $j$ below. Using $z^{k}(1-z)=p^{k}(1-p)$, we obtain

$$
\frac{d z}{d p}=\frac{p^{k-1}}{z^{k-1}} \frac{p-p_{*}}{z-p_{*}} .
$$

Recall $p_{*}=k /(k+1)$ and $q_{*}=1 /(k+1)$. Then

$$
\begin{aligned}
\frac{d(z \bar{z})}{d p} & =\frac{p^{k-1}}{z^{k-1}} \frac{p-p_{*}}{z-p_{*}} \bar{z}+\frac{p^{k-1}}{\bar{z}^{k-1}} \frac{p-p_{*}}{\bar{z}-p_{*}} z \\
& \equiv\left(p-p_{*}\right) \frac{p^{k-1}}{|z|^{2(k-1)}} \frac{\mathcal{N}}{\left(z-p_{*}\right)\left(\bar{z}-p_{*}\right)}
\end{aligned}
$$

The denominator $|z|^{2(k-1)}\left(z-p_{*}\right)\left(\bar{z}-p_{*}\right)$ never vanishes for $p \in(0,1)$. The numerator $\mathcal{N}$, which is real, can be simplified as follows:

$$
\begin{aligned}
\mathcal{N} & =z^{k}\left(z-1+q_{*}\right)+\bar{z}^{k}\left(\bar{z}-1+q_{*}\right) \\
& =q_{*}\left(z^{k}+\bar{z}^{k}\right)-2 p^{k} q<2 q_{*}|z|^{k}-2 p^{k} q \\
& =2 p^{k} q \frac{q_{*}-|1-z|}{|1-z|}<0 .
\end{aligned}
$$

The last line follows because $|z|<\min \{p, \alpha\} \leq p_{*}$; hence $|1-z|>1-|z|>1-p_{*}=q_{*}$. The inequalities are strict because $z$ is not real and positive. Hence $\mathcal{N} \neq 0$ and $z \bar{z}$ attains an extremum only if the factor $\left(p-p_{*}\right)$ vanishes; that is, $p=$ $k /(k+1)$. The extremum is obviously a maximum.

Remark 18 (moment generating function). An expression for the probability generating function $\mathscr{P}(s)$ was derived by Feller [9] (see (3)) and rederived by Philippou et al. [3]. An equivalent expression for the moment generating function $M(t)=\mathscr{P}\left(e^{t}\right)=\mathbb{E}\left[e^{t X}\right]$ was derived by Barry and Lo Bello [13]. Feller stated that the domain of convergence of $\mathscr{P}(s)$ is $|s|<1 / \alpha$, whereas Philippou et al. stated that $\mathscr{P}(s)$ exists for $|s| \leq 1$. Barry and Lo Bello denoted the roots by $z_{j}, j=1, \ldots, k$, and defined $m=$ $\min \left\{-\ln \left|z_{1}\right|,-\ln \left|z_{2}\right|, \ldots,-\ln \left|z_{k}\right|\right\}$. They proved that all the roots have modulus less than unity and stated that $M(t)$ exists on the interval $t \in(-\infty, m)$. We confirm the correctness of Feller's statement that the domain of convergence is determined by the principal root; hence $|s|<1 / \alpha$ yields the most precise value for the domain of convergence.

The following expressions for the mean $\mu$ and variance $\sigma^{2}$ of the waiting time for the first run of $k$ successes were derived by Feller [9] (and rederived by Philippou et al. [3]):

$$
\begin{aligned}
\mu & =\frac{1-p^{k}}{q p^{k}}, \\
\sigma^{2} & =\frac{1}{\left(q p^{k}\right)^{2}}-\frac{2 k+1}{q p^{k}}-\frac{p}{q^{2}} .
\end{aligned}
$$

Chaves and de Souza [14] obtained the above expression for the mean but a different expression for the variance. We confirm the correctness of Feller's expressions. The mean and variance are polynomials in $1 / p$ and it is easily derived that

$$
\begin{aligned}
\mu & =\frac{1}{p}+\frac{1}{p^{2}}+\cdots+\frac{1}{p^{k}} \\
\sigma^{2} & =\frac{1-p}{2}\left[\frac{k(k+1)}{p^{k+1}}\right. \\
& \left.+\sum_{j=0}^{k-2}(j+1)(j+2)\left(\frac{1}{p^{2 k-j}}+\frac{1}{p^{2+j}}\right)\right] .
\end{aligned}
$$

From the above we see that both the mean and variance decrease strictly and continuously with $p$. In particular $\mu=k$ and $\sigma^{2}=0$ at $p=1$, as expected. Both $\mu$ and $\sigma^{2}$ diverge as $p \rightarrow 0$.

\section{Power Series Solutions for Roots}

We now derive expressions for the roots as sums of power series. We begin with the principal root $\alpha$. We already know that $\alpha=k /(k+1)$ when $p=k /(k+1)$. Next we treat the case $k /(k+1)<p<1$, so $0<\alpha<k /(k+1)$. Let $F(z):=$ $z(1-z)^{1 / k}, D_{1}:=\{z:|z|<k /(k+1)\}$, and $D_{2}:=\{w:|w|<$ $\left.k /(k+1)^{(k+1) / k}\right\}$. 
Proposition 19. $F$ is a conformal homeomorphism from $U:=$ $D_{1} \cap F^{-1}\left(D_{2}\right)$ onto $D_{2}$.

Proof. If $|z|=k /(k+1)$ then $|F(z)| \geq k /(k+1)^{(k+1) / k}$, and hence $D_{2} \subset f\left(D_{1}\right)$. Thus it suffices to show that $F$ is oneone on $U$. Note that $F^{\prime}(z)=(1-z)^{1 / k-1}(k-(k+1) z) / k$. So $F^{\prime}(z) \neq 0$ for all $z \in D_{1}$, which implies that $F$ is locally oneone on $D_{1}$. Let

$$
A:=\left\{w \in D_{2}: \exists z, \zeta \in U, z \neq \zeta, F(z)=F(\zeta)=w\right\},
$$

and suppose, to derive a contradiction, that $A$ is nonempty. Let $\rho_{0}:=\inf \{|w|: w \in A\}$. Suppose that $\rho_{0}=0$. Then there exist $\left(z_{n}\right),\left(\zeta_{n}\right) \subset U$ such that $z_{n} \neq \zeta_{n}$ and $F\left(z_{n}\right)=F\left(\zeta_{n}\right) \rightarrow 0$. Clearly, $z_{n} \rightarrow 0$ and $\zeta_{n} \rightarrow 0$. But this contradicts the fact that $F$ is one-one on a neighborhood of $z=0$. So $0<\rho_{0}<$ $k /(k+1)^{(k+1) / k}$. There exist $\left(z_{n}\right),\left(\zeta_{n}\right) \subset U$ and $\left(w_{n}\right) \subset D_{2}$ such that $z_{n} \neq \zeta_{n}, F\left(z_{n}\right)=F\left(\zeta_{n}\right)=w_{n}$, and $\left|w_{n}\right| \rightarrow \rho_{0}$. Passing to a subsequence, we may assume that $z_{n} \rightarrow z_{0}, \zeta_{n} \rightarrow \zeta_{0}$, and $w_{n} \rightarrow w_{0}$. Since $\rho_{0}<k /(k+1)^{(k+1) / k}$, it follows that there exists $r<k /(k+1)$ such that $\left|z_{n}\right|,\left|\zeta_{n}\right| \leq r$ for all $n$. So $\left|z_{0}\right|,\left|\zeta_{0}\right| \leq r$, and hence $z_{0}, \zeta_{0} \in D_{1}$. By continuity $F\left(z_{0}\right)=$ $f\left(\zeta_{0}\right)=w_{0} \in D_{2}$, so $z_{0}, \zeta_{0} \in U$. Moreover, $\left|w_{0}\right|=\lim \left|w_{n}\right|=$ $\rho_{0}$. Since $F$ is one-one on a neighborhood of $z_{0}$ it follows that $\zeta_{0} \neq z_{0}$. Choose disjoint open neighborhoods $N_{1} \subset U$ of $z_{0}$ and $N_{2} \subset U$ of $\zeta_{0}$. By the Open Mapping Theorem $F\left(N_{1}\right) \cap$ $F\left(N_{2}\right)$ is an open neighborhood of $w_{0}$. So there exist $z^{\prime} \in N_{1}$, $\zeta^{\prime} \in N_{2}$ such that $F\left(z^{\prime}\right)=F\left(\zeta^{\prime}\right)=w^{\prime}$, where $\left|w^{\prime}\right|<\left|w_{0}\right|=\rho_{0}$. Since $z^{\prime} \neq \zeta^{\prime}$, this contradicts the definition of $\rho_{0}$ and hence contradicts the assumption that $A$ is nonempty.

Since $F$ is a conformal homeomorphism its inverse $z:=$ $G(w)$ is analytic on $D_{2}$. Hence $G(w)=\sum_{n=1}^{\infty} b_{n} w^{n}$ for $w \in D_{2}$ for some scalar sequence $\left(b_{n}\right)_{n=1}^{\infty}$ (we show in Proposition 23 below that $b_{n}$ is given by (6)). Note that $k /(k+1) \in \bar{U}$ but $F^{\prime}(k /(k+1))=0$. Hence the functional equation $G(F(z))=z$ cannot be continued through $z=k /(k+1)$. It follows that $G(z)$ cannot be continued analytically through $w=k /(k+$ $1)^{(k+1) / k}$. In particular, the Taylor series for $G$ has radius of convergence $k /(k+1)^{(k+1) / k}$.

Corollary 20. Suppose $k /(k+1)<p<1$. Then

$$
\alpha=G\left(p q^{1 / k}\right)=\sum_{n=1}^{\infty} b_{n}\left(p q^{1 / k}\right)^{n}
$$

Proof. Note that $\alpha<k /(k+1)$ since $p>k /(k+1)$ and that $F(\alpha)=\alpha(1-\alpha)^{1 / k}=p q^{1 / k}$. Hence $G\left(p q^{1 / k}\right)=G(F(\alpha))=$ $\alpha$.

Corollary 21. For all $p \in(0,1)$, the secondary roots are given by (note that $\lambda_{j}=\overline{\lambda_{k-j}}$ )

$$
\lambda_{j}=G\left(e^{2 \pi i j / k} p q^{1 / k}\right) \quad(1 \leq j \leq k-1) .
$$

Proof. First suppose $p \neq k /(k+1)$. Then $p q^{1 / k} e^{2 \pi i j / k} \in D_{2}$ for $1 \leq j \leq k-1$. So for each $1 \leq j \leq k-1$ there exist distinct $\lambda_{j} \in D_{1}$ such that $F\left(\lambda_{j}\right)=p q^{1 / k} e^{2 \pi i j / k}$. Thus, $\lambda_{j}=$ $G\left(F\left(\lambda_{j}\right)\right)=G\left(p q^{1 / k} e^{2 \pi i j / k}\right)$, and

$$
\lambda_{j}^{k}\left(1-\lambda_{j}\right)=F\left(\lambda_{j}\right)^{k}=\left(p q^{1 / k} e^{2 \pi i j / k}\right)^{k}=p^{k} q,
$$

so $\lambda_{j}$ is a secondary root as desired. The power series for $G(w)$ has positive coefficients (see Proposition 23 below) and converges at $w=p q^{1 / k}$ when $p=k /(k+1)$. Hence $G$ extends continuously to $\overline{D_{2}}$ and the series for each $\lambda_{j}$ converges absolutely when $p=k /(k+1)$. By continuity of $G$ the series for each $\lambda_{j}$, when $p=k /(k+1)$, represents a secondary root of the auxiliary equation as desired.

Corollary 22. For $0<p<k /(k+1)$, the principal root is given by

$$
\alpha=1-k \sum_{m=1}^{\infty} b_{m k}\left(p^{k} q\right)^{m} .
$$

Proof. The sum of the roots is $q$; hence the secondary roots sum up to $q-\alpha$. Using $\alpha(1-\alpha)^{1 / k}=p q^{1 / k}$, it follows that $G\left(p q^{1 / k}\right)=\min \{p, \alpha\}$ for all $p \in(0,1)$. Hence $G\left(p q^{1 / k}\right)=p$ for $p \in(0, k /(k+1))$ and so

$$
\sum_{j=0}^{k-1} G\left(e^{2 \pi i j / k} p q^{1 / k}\right)=p+q-\alpha=1-\alpha .
$$

Hence

$$
\begin{aligned}
\alpha & =1-\sum_{j=0}^{k-1} \sum_{n=1}^{\infty} b_{n}\left(e^{2 \pi i j / k} p q^{1 / k}\right)^{n} \\
& =1-\sum_{n=1}^{\infty} b_{n}\left(p q^{1 / k}\right)^{n} \sum_{j=0}^{k-1}\left(e^{2 \pi i j / k}\right)^{n} \\
& =1-\sum_{k \mid n}^{\infty} b_{n} k\left(p q^{1 / k}\right)^{n}=1-k \sum_{m=1}^{\infty} b_{m k}\left(p^{k} q\right)^{m} .
\end{aligned}
$$

In the second line, the sum over the roots of unity vanishes unless $n$ is a multiple of $k$. The interchange of the orders of summation is permissible because the series converge absolutely within the domain of convergence.

Proposition 23. The coefficients $b_{n}$ are given by

$$
b_{n}=\frac{\Gamma(n-1+n / k)}{n ! \Gamma(n / k)} .
$$

Asymptotically for large $n$ (this is an application of Stirling's formula and the proof is omitted)

$$
b_{n}=\frac{1}{(2 \pi)^{1 / 2}} \frac{k}{(k+1)^{3 / 2}} \frac{1}{n^{3 / 2}}\left(\frac{(k+1)^{(k+1) / k}}{k}\right)^{n} .
$$

Proof. From (41), and $\alpha(1-\alpha)^{1 / k}=p q^{1 / k}$,

$$
\alpha=\sum_{n=1}^{\infty} b_{n} \alpha^{n}(1-\alpha)^{n / k} .
$$


Then $b_{1}=1$ and $b_{2}=1 / k$ are easily derived. In the manipulations below, it is convenient to employ the falling factorial $(x)_{r}$ and rising factorial $x^{(r)}$, where $(x)_{0}=x^{(0)}=1$, while, for $r \geq 1,(x)_{r}=x(x-1) \cdots(x-r+1)$ and $x^{(r)}=$ $x(x+1) \cdots(x+r-1)$. We obtain $b_{n+1}$ for $n \geq 2$ by equating terms in $\alpha^{n+1}$

$$
0=\sum_{m=1}^{n+1}(-1)^{n-m} b_{m} \frac{(m / k)_{n-m+1}}{(n-m+1) !} .
$$

The coefficient of $b_{n+1}$ is -1 , so by rearranging terms we can obtain $b_{n+1}$ in terms of $b_{1}, \ldots, b_{n}$. We thus formulate the following claim: we claim that $\chi=0$ identically, where

$$
\chi=k n ! \sum_{m=1}^{n+1}(-1)^{m-1} \frac{(m / k)_{n-m+1} \Gamma(m-1+m / k)}{(n-m+1) ! m ! \Gamma(m / k)} .
$$

Next note that $\Gamma(m-1+m / k) / \Gamma(m / k)=(m / k)^{(m-1)}$. Hence for all $1 \leq m \leq n+1$, we obtain the following product of $m / k$ and $n-1$ consecutive terms:

$$
\begin{aligned}
& \frac{\Gamma(m-1+m / k)}{\Gamma(m / k)}\left(\frac{m}{k}\right)_{n-m+1}=\left(\frac{m}{k}\right)^{(m-1)}\left(\frac{m}{k}\right)_{n-m+1} \\
& =\left(\frac{m}{k}\right)\left(-2+m+\frac{m}{k}\right)_{n-1} \\
& =\frac{m}{k} \prod_{r=2}^{n}\left(-r+m+\frac{m}{k}\right) .
\end{aligned}
$$

Hence

$$
\chi=\sum_{m=1}^{n+1}(-1)^{m-1}\left(\begin{array}{c}
n \\
m-1
\end{array}\right) \prod_{r=2}^{n}\left(-r+m+\frac{m}{k}\right) .
$$

For convenience below, replace $1+1 / k$ by $x \in \mathbb{R}$. Also define $m^{\prime}=m-1$ so $0 \leq m^{\prime} \leq n$. Then, dropping the prime,

$$
\chi(x)=\sum_{m=0}^{n}(-1)^{m}\left(\begin{array}{l}
n \\
m
\end{array}\right) \prod_{r=2}^{n}(-r+(m+1) x) .
$$

The product is a polynomial of degree $n-1$ in $(m+1) x$ :

$$
\prod_{r=2}^{n}(-r+(m+1) x)=\sum_{s=0}^{n-1} \widetilde{c}_{s}(n)(m+1)^{s} x^{s} .
$$

Note that the coefficients $\widetilde{c}_{s}(n)$ depend on $n$ but not on $m$. (e.g., $\widetilde{c}_{0}=(-1)^{n-1} n !$.) Hence we can write

$$
\chi(x)=\sum_{s=0}^{n-1} \widetilde{c}_{s}(n) x^{s}\left[\sum_{m=0}^{n}(-1)^{m}\left(\begin{array}{l}
n \\
m
\end{array}\right)(m+1)^{s}\right] .
$$

It is a standard identity of binomial coefficients that, for all $0 \leq s \leq n-1$,

$$
\sum_{m=0}^{n}(-1)^{m}\left(\begin{array}{l}
n \\
m
\end{array}\right) m^{s}=0 .
$$

(Essentially, expand $(1-x)^{n}$ in a binomial series and differentiate $s=0,1, \ldots, n-1$ times and evaluate the sum at $x=1$.) It follows that $\chi(x)=0$ identically. Setting $x=1+1 / k$ then yields the original claim.

\section{The Probability Mass Function}

From the theory of recurrence relations, there exist constants $c_{j}(p, k), j=0,1, \ldots, k-1$, such that

$$
f_{k}(n)=\sum_{j=0}^{k-1} c_{j} \lambda_{j}^{n-1}
$$

We omit the dependences on $k$ and $p$ in (58) and below. Recall the initial conditions are $f_{k}(n)=0$ for $n=1, \ldots, k-1$ and $f_{k}(k)=p^{k}$. The equations to solve for the $c_{j}$ form a Vandermonde system:

$$
\begin{gathered}
\left(\begin{array}{cccc}
1 & 1 & \cdots & 1 \\
\lambda_{0} & \lambda_{1} & \cdots & \lambda_{k-1} \\
\vdots & & & \vdots \\
\lambda_{0}^{k-2} & \lambda_{1}^{k-2} & \cdots & \lambda_{k-1}^{k-2} \\
\lambda_{0}^{k-1} & \lambda_{1}^{k-1} & \cdots & \lambda_{k-1}^{k-1}
\end{array}\right)\left(\begin{array}{c}
c_{0} \\
c_{1} \\
\vdots \\
c_{k-2} \\
c_{k-1}
\end{array}\right) \\
=\left(\begin{array}{c}
0 \\
0 \\
\vdots \\
0 \\
p^{k}
\end{array}\right) .
\end{gathered}
$$

It is then known, from the properties of Vandermonde matrices, that the solution is

$$
c_{j}=\frac{p^{k}}{\prod_{m=0,1, \ldots, k-1, m \neq j}\left(\lambda_{j}-\lambda_{m}\right)} .
$$

This can be expressed more concisely as follows. Note that the auxiliary polynomial $\mathscr{A}(z)$ can be written as

$$
\mathscr{A}(z)=\left(z-\lambda_{0}\right)\left(z-\lambda_{1}\right) \cdots\left(z-\lambda_{k-1}\right) .
$$

Hence for $j=0,1, \ldots, k-1$,

$$
\mathscr{A}^{\prime}\left(\lambda_{j}\right)=\prod_{m=0,1, \ldots, k-1, m \neq j}\left(\lambda_{j}-\lambda_{m}\right) .
$$

Hence $c_{j}=p^{k} / \mathscr{A}^{\prime}\left(\lambda_{j}\right)$. Next note that $\mathscr{B}^{\prime}(z)=(z-p) \mathscr{A}^{\prime}(z)+$ $\mathscr{A}(z)$. Then provided $\lambda_{j} \neq p$ (which can happen only if $p=k /(k+1)$, and only for the positive root) $\mathscr{B}^{\prime}\left(\lambda_{j}\right)=$ $\left(\lambda_{j}-p\right) \mathscr{A}^{\prime}\left(\lambda_{j}\right)$. From (5), $\mathscr{B}^{\prime}\left(\lambda_{j}\right)=(k+1) \lambda_{j}^{k}-k \lambda_{j}^{k-1}$. Hence for $\lambda_{j} \neq p$,

$$
\mathscr{A}^{\prime}\left(\lambda_{j}\right)=(k+1) \lambda_{j}^{k-1} \frac{\lambda_{j}-k /(k+1)}{\lambda_{j}-p} .
$$

Hence

$$
c_{j} \lambda_{j}^{n-1}=\frac{p^{k} \lambda_{j}^{n-k}}{k+1} \frac{\lambda_{j}-p}{\lambda_{j}-k /(k+1)} .
$$

Substitution into (58) yields (15) for $p \neq k /(k+1)$. 
Next we treat the case $\lambda_{j}=p$. This requires $\lambda_{j}$ to be real and positive; that is, $\lambda_{j}=\alpha$, and we know $\alpha=p$ if and only if $p=k /(k+1)$. Hence we set $j=0$ and $\lambda_{0}=\alpha$ in (63). The term $\alpha^{k-1}$ is finite and positive for all $p \in(0,1)$. To evaluate the ratio in (63), which is a $0 / 0$ indeterminate form, we expand about $k /(k+1)$. Let $p=p_{*}+\delta$ and set $z=p_{*}+\epsilon$ in (5), recalling that $p_{*}=k /(k+1), q_{*}=1 /(k+1)$, and $p_{*}=k q_{*}$. Hence

$$
\begin{aligned}
0 & =\left(p_{*}+\epsilon\right)^{k}\left(\epsilon-q_{*}\right)-\left(p_{*}+\delta\right)^{k}\left(\delta-q_{*}\right) \\
& \simeq \frac{k p_{*}^{k-2}}{2}\left(\epsilon^{2}-\delta^{2}\right) .
\end{aligned}
$$

Since $\epsilon \neq \delta$, the solution is $\epsilon \simeq-\delta$. Then (63) yields

$$
\mathscr{A}^{\prime}(\alpha)=\lim _{\delta \rightarrow 0}(k+1)\left(p_{*}+\epsilon\right)^{k-1} \frac{\epsilon}{\epsilon-\delta}=\frac{k+1}{2} p_{*}^{k-1} .
$$

For all the secondary roots, if $p=p_{*}$ then (63) yields

$$
\mathscr{A}^{\prime}\left(\lambda_{j}\right)=(k+1) \lambda_{j}^{k-1} .
$$

Hence for $p=k /(k+1)$,

$$
\begin{aligned}
& c_{0} \lambda_{0}^{n-1}=\frac{2 p_{k} \alpha^{n-k}}{k+1}, \\
& c_{j} \lambda_{j}^{n-1}=\frac{p_{k} \lambda_{j}^{n-k}}{k+1}
\end{aligned}
$$

$$
(1 \leq j \leq k-1) .
$$

Substitution into (58) yields (15) for $p=k /(k+1)$.

The derivation of the asymptotic expressions in Corollary 4 goes as follows. Because the principal root has a strictly larger magnitude than all the other roots, for sufficiently large $n$ it dominates the contribution to $f_{k}(n)$. From (15) for $p \neq k /(k+1)$,

$$
\begin{aligned}
f_{k}(n) & =\frac{p^{k} \alpha^{n-k}}{k+1} \frac{\alpha-p}{\alpha-k /(k+1)} \\
& =\alpha^{n} \frac{(1-\alpha)(\alpha-p)}{((k+1) \alpha-k) q} .
\end{aligned}
$$

In the last line we used $p^{k} q=\alpha^{k}(1-\alpha)$. The expression in (69) agrees with that by Feller [9]. Next for $p=k /(k+1)$, using $\alpha=p$,

$$
f_{k}(n)=\frac{2 p^{k} \alpha^{n-k}}{k+1}=\frac{2 p^{n}}{k+1} .
$$

This expression is not in [9].

\section{Asymptotics}

We derive (17) and (18). For given fixed $\epsilon>0$, we want $N(p, k)$ such that, for all $n \geq N$, the magnitude of the summed contribution from all the secondary roots in (15) is less than $\epsilon$ times the contribution from the principal root. From (15), one must have

$$
\left|\sum_{j=1}^{k-1}\left(\frac{\lambda_{j}}{\alpha}\right)^{N-k} \frac{\lambda_{j}-p}{\lambda_{j}-k /(k+1)}\right|<\epsilon \frac{\alpha-p}{\alpha-k /(k+1)} .
$$

Now suppose that we have an upper bound $\kappa(p, k)$ such that $\left|\lambda_{j} / \alpha\right|<\kappa<1$ for all the secondary roots. Note that $\kappa$ depends on $p$ and $k$ but not on $j$. The contribution from the secondary roots is bounded by

$$
\begin{gathered}
\left|\sum_{j=1}^{k-1}\left(\frac{\lambda_{j}}{\alpha}\right)^{N-k} \frac{\lambda_{j}-p}{\lambda_{j}-k /(k+1)}\right| \\
\quad<\sum_{j=1}^{k-1}\left|\frac{\lambda_{j}}{\alpha}\right|^{N-k}\left|\frac{\lambda_{j}-p}{\lambda_{j}-k /(k+1)}\right| \\
\quad<\kappa^{N-k} \sum_{j=1}^{k-1}\left|\frac{\lambda_{j}-p}{\lambda_{j}-k /(k+1)}\right| .
\end{gathered}
$$

Hence from (71) the contribution of the principal root will dominate if

$$
\kappa^{N-k} \sum_{j=1}^{k-1}\left|\frac{\lambda_{j}-p}{\lambda_{j}-k /(k+1)}\right|<\epsilon \frac{\alpha-p}{\alpha-k /(k+1)} .
$$

In the special case $p=k /(k+1)$, we see from (15) that (73) simplifies to

$$
2 \epsilon>\sum_{j=1}^{k-1} \kappa^{N-k}=(k-1) \kappa^{N-k} .
$$

Hence we require $\kappa^{N-k}<2 \epsilon /(k-1)$. Next if $0<p<k /(k+1)$, then $k /(k+1)<\alpha<1$ and $\Re\left(\lambda_{j}\right)<p$, so

$$
\left|\frac{\lambda_{j}-p}{\lambda_{j}-k /(k+1)}\right|<1<\frac{\alpha-p}{\alpha-k /(k+1)} .
$$

Hence in (73)

$$
\epsilon>\kappa^{N-k} \sum_{j=1}^{k-1} 1=(k-1) \kappa^{N-k} .
$$

Hence we require $\kappa^{N-k}<\epsilon /(k-1)$. Next if $k /(k+1)<p<1$, then $0<\alpha<k /(k+1)$. Note that because $\mathfrak{R}\left(\lambda_{j}\right)<\alpha<$ $k /(k+1)<p$, it follows that

$$
\left|\frac{\lambda_{j}-p}{\lambda_{j}-k /(k+1)}\right|<\frac{\alpha-p}{\alpha-k /(k+1)} .
$$

Hence (73) yields

$$
\begin{aligned}
\epsilon \frac{\alpha-p}{\alpha-k /(k+1)} & >\kappa^{N-k} \sum_{j=1}^{k-1} \frac{\alpha-p}{\alpha-k /(k+1)} \\
& =\frac{\alpha-p}{\alpha-k /(k+1)}(k-1) \kappa^{N-k} .
\end{aligned}
$$


Hence the condition is again $\kappa^{N-k}<\epsilon /(k-1)$. Hence for all $p \in(0,1)$, a sufficient condition is $\kappa^{N-k}=\epsilon /(k-1)$. Taking logarithms yields (17); namely,

$$
N=k+\frac{\ln (\epsilon /(k-1))}{\ln \kappa} .
$$

The task then is to determine $\kappa$. We proved that $\left|\lambda_{j}\right|<p$ for all the secondary roots and $\alpha>q$. Hence a possible bound is

$$
\frac{\left|\lambda_{j}\right|}{\alpha}<\frac{p}{1-p} .
$$

This is not a tight upper bound; it is meaningful only if $p<$ $1-p$; that is, $p<1 / 2$. Next if $0<p<k /(k+1)$, so $k /(k+1)<$ $\alpha<1$; we see that

$$
\frac{\left|\lambda_{j}\right|}{\alpha}<\frac{p(k+1)}{k} .
$$

Next we need an estimate for $\kappa$ for $k /(k+1) \leq p<1$.

Lemma 24. For $p \in[k /(k+1), 1), \kappa=1-\delta$, where

$$
\begin{aligned}
\delta & =\frac{1}{(k+1)^{1 / k}}\left[1+\frac{p q^{1 / k}}{k}\right. \\
& \left.-\sqrt{1+\frac{\left(p q^{1 / k}\right)^{2}}{k^{2}}+\frac{2 p q^{1 / k}}{k} \cos \left(\frac{2 \pi}{k}\right)}\right] .
\end{aligned}
$$

Proof. Let $\omega_{k}=e^{2 \pi i / k}$. Suppose $p \geq k /(k+1)$, so $\alpha \leq k /(k+1)$. Consider the roots $z_{j}=G\left(\omega_{k}^{j} p q^{1 / k}\right), 0 \leq j<k$. Then $z_{0}=\alpha$. We show that, for $j=1, \ldots, k-1,\left|z_{j}\right|<(1-\delta) \alpha$ for some $\delta(k, p)>0$ which we compute. Then for $j \geq 1$, because $b_{n}>0$, for all $n$,

$$
\begin{aligned}
\left|z_{j}\right| & =\left|G\left(\omega_{k}^{j} p q^{1 / k}\right)\right| \\
& \leq\left|\omega_{k}^{j} p q^{1 / k}+\frac{\left(\omega_{k}^{j} p q^{1 / k}\right)^{2}}{k}\right|+\sum_{n=3}^{\infty} b_{n}\left(p q^{1 / k}\right)^{n} \\
& =p q^{1 / k}\left|1+\frac{\left(\omega_{k}^{j} p q^{1 / k}\right)}{k}\right|+\sum_{n=3}^{\infty} b_{n}\left(p q^{1 / k}\right)^{n} .
\end{aligned}
$$

Then because $\left|1+\left(\omega_{k}^{j} p q^{1 / k}\right) / k\right|$ is maximized at $j=1$,

$$
\begin{aligned}
\left|z_{j}\right| \leq & p q^{1 / k}\left|1+\frac{\left(\omega_{k} p q^{1 / k}\right)}{k}\right|+\sum_{n=3}^{\infty} b_{n}\left(p q^{1 / k}\right)^{n} \\
= & p q^{1 / k}\left|1+\frac{\left(\omega_{k} p q^{1 / k}\right)}{k}\right|+G\left(p q^{1 / k}\right)-p q^{1 / k} \\
& -\frac{\left(p q^{1 / k}\right)^{2}}{k} .
\end{aligned}
$$

Next recall $G\left(p q^{1 / k}\right)=\alpha$ and $p q^{1 / k}=\alpha(1-\alpha)^{1 / k}$, so substituting into the last expression

$$
\begin{aligned}
\frac{\left|z_{j}\right|}{\alpha} & \leq 1-(1-\alpha)^{1 / k}\left(1+\frac{p q^{1 / k}}{k}-\left|1+\frac{\left(\omega_{k} p q^{1 / k}\right)}{k}\right|\right) \\
& \leq 1-\frac{1}{(k+1)^{1 / k}}\left(1+\frac{p q^{1 / k}}{k}\right. \\
- & \left.\sqrt{1+\frac{\left(p q^{1 / k}\right)^{2}}{k^{2}}+\frac{2 p q^{1 / k}}{k} \cos \left(\frac{2 \pi}{k}\right)}\right) .
\end{aligned}
$$

The last line follows because $\alpha \leq k /(k+1)$. Thus we can take $\delta$ as defined in (82).

The bound $\kappa=1-\delta$ using (82) is also applicable for $p \epsilon$ $(0, k /(k+1))$; to see this use $\min \{p, \alpha\}$ in the derivation above. Then for all $p \in(0,1)$, we select the most stringent bound at each value of $p$. This yields the various cases in (18).

\section{Conflict of Interests}

The authors declare that there is no conflict of interests regarding the publication of this paper.

\section{Acknowledgments}

The authors thank Dr. P. M. Strickland and Professor K. Yokoya for their helpfulness. This material is based upon work of the first author, who was supported by the National Science Foundation under Grant no. DMS-1361461.

\section{References}

[1] N. Balakrishnan and M. V. Koutras, Runs and Scans with Applications, Wiley, New York, NY, USA, 2002.

[2] N. L. Johnson, A. W. Kemp, and S. Kotz, Univariate Discrete Distributions, Wiley-Interscience, Hoboken, NJ, USA, 3rd edition, 2005.

[3] A. N. Philippou, C. Georghiou, and G. N. Philippou, "A generalized geometric distribution and some of its properties," Statistics \& Probability Letters, vol. 1, no. 4, pp. 171-175, 1983.

[4] A. N. Philippou and A. A. Muwafi, "Waiting for the $k^{\text {th }}$ consecutive success and the fibonacci sequence of order $k$," The Fibonacci Quarterly, vol. 20, no. 1, pp. 28-32, 1982.

[5] E. J. Burr and G. Cane, "Longest run of consecutive observations having a specified attribute," Biometrika, vol. 48, pp. 461-465, 1961.

[6] A. P. Godbole, "Specific formulae for some success run distributions," Statistics \& Probability Letters, vol. 10, no. 2, pp. 119-124, 1990.

[7] A. N. Philippou and F. S. Makri, "Longest success runs and Fibonacci-type polynomials," The Fibonacci Quarterly, vol. 23, no. 4, pp. 338-346, 1985. 
[8] M. Muselli, "Simple expressions for success run distributions in Bernoulli trials," Statistics \& Probability Letters, vol. 31, no. 2, pp. 121-128, 1996.

[9] W. Feller, An Introduction to Probability Theory and its Applications, Wiley, New York, NY, USA, 3rd edition, 1968.

[10] R. A. Graham, D. E. Knuth, and O. Patashnik, Concrete Mathematics, Addison-Wesley, New York, NY, USA, 2nd edition, 1994.

[11] S. Eryilmaz, "Geometric distribution of order $k$ with a reward," Statistics \& Probability Letters, vol. 92, pp. 53-58, 2014.

[12] E. Shmerling, "Cumulative distribution function of Markov order 2 geometric distribution of order k," Functional Differential Equations, vol. 20, no. 1-2, pp. 129-137, 2013.

[13] M. J. J. Barry and A. Lo Bello, "The moment generating function of the geometric distribution of order $k$," The Fibonacci Quarterly, vol. 31, no. 2, pp. 178-180, 1993.

[14] L. M. Chaves and D. J. de Souza, "Waiting time for a run of $\mathrm{N}$ successes in Bernoulli sequences," Revista Brasileira de Biomedicina, vol. 25, pp. 101-113, 2007. 


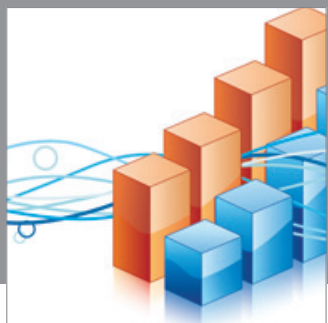

Advances in

Operations Research

mansans

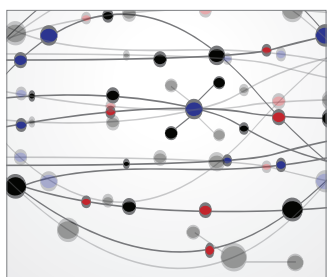

The Scientific World Journal
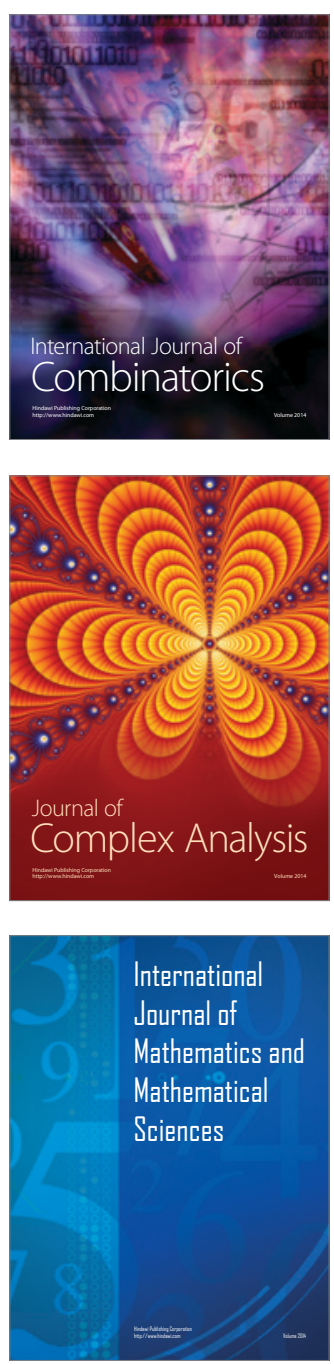
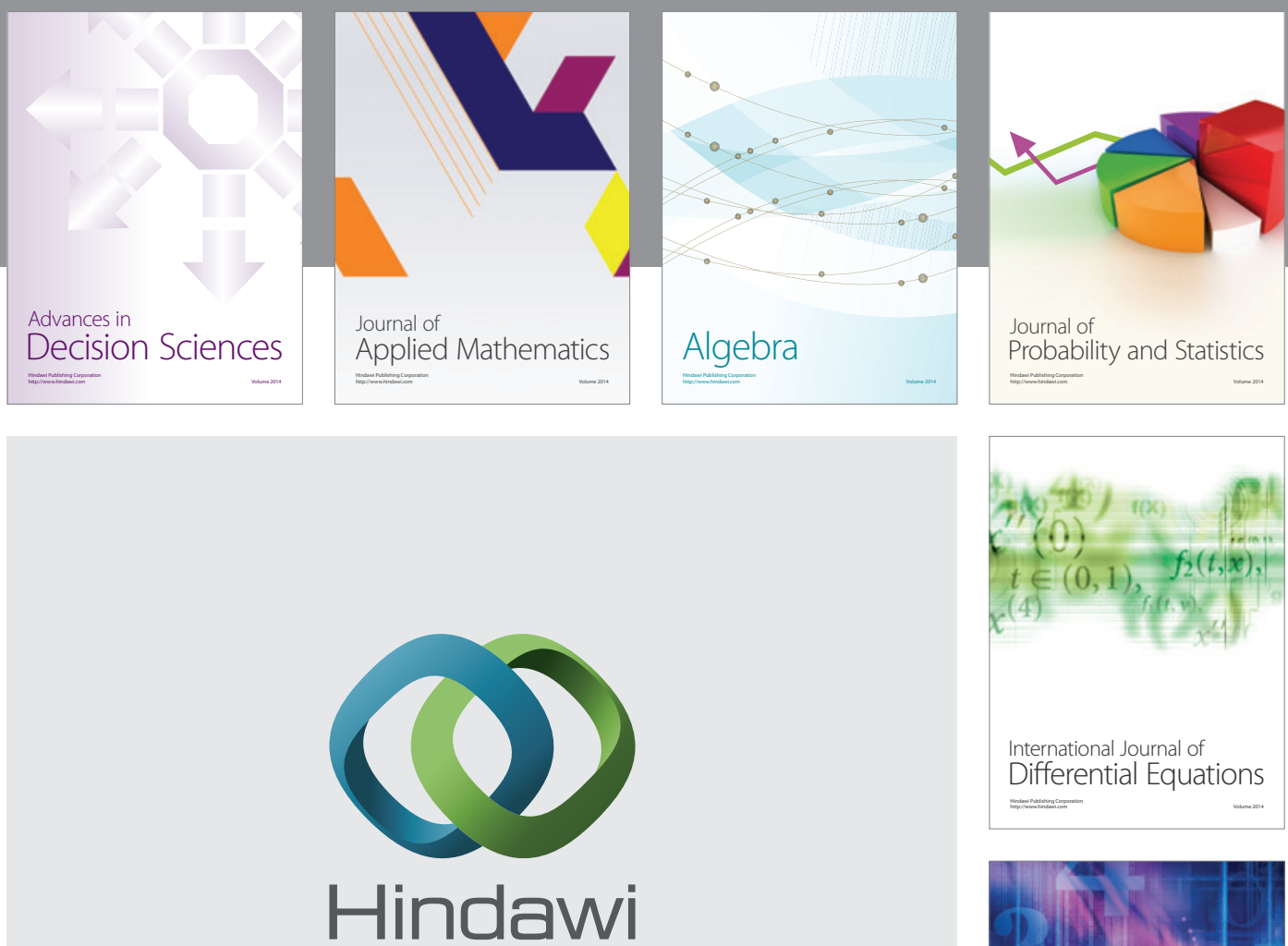

Submit your manuscripts at http://www.hindawi.com
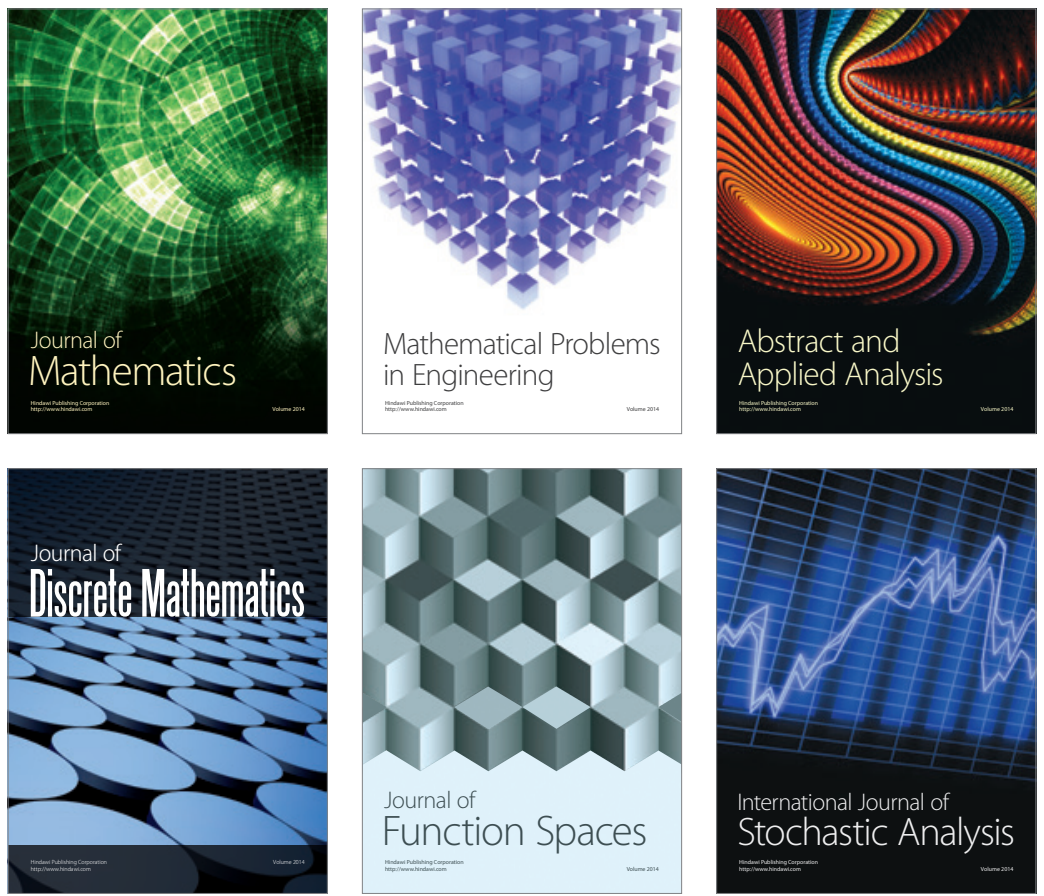

Journal of

Function Spaces

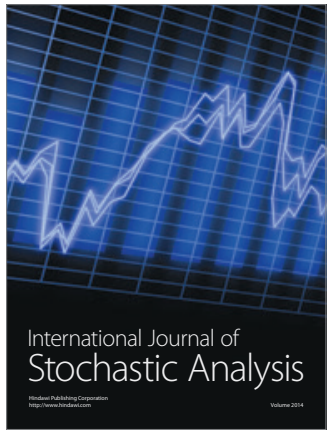

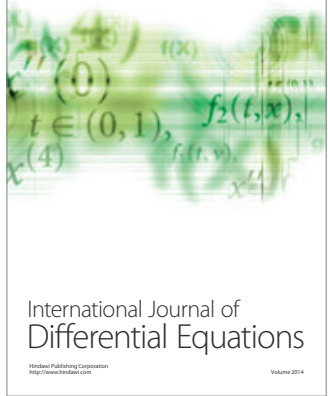
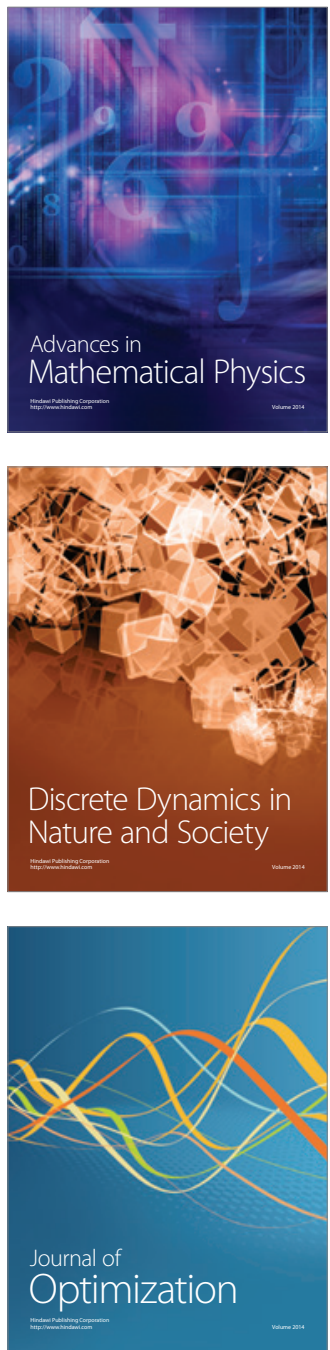